\title{
Quantifying Single mRNA Translation Kinetics in Living Cells
}

\section{Tatsuya Morisaki ${ }^{1}$ and Timothy J. Stasevich ${ }^{1,2}$}

\author{
${ }^{1}$ Institute of Genome Architecture and Function and Department of Biochemistry \& Molecular Biology, Colorado State \\ University, Fort Collins, Colorado 80523 \\ ${ }^{2}$ Cell Biology Unit, Institute of Innovative Research, Tokyo Institute of Technology, Midori-ku, Yokohama, Kanagawa, \\ 226-8503, Japan
}

Correspondence: tim.stasevich@colostate.edu; tatsuya.morisaki@colostate.edu

\section{SUMMARY}

One of the last hurdles to quantifying the full central dogma of molecular biology in living cells with single-molecule resolution has been the imaging of single messenger RNA (mRNA) translation. Here we describe how recent advances in protein tagging and imaging technologies are being used to precisely visualize and quantify the synthesis of nascent polypeptide chains from single mRNA in living cells. We focus on recent applications of repeat-epitope tags and describe how they enable quantification of single mRNA ribosomal densities, translation initiation and elongation rates, and translation site mobility and higher-order structure. Together with complementary live-cell assays to monitor translation using fast-maturing fluorophores and mRNA-binding protein knockoff, single-molecule studies are beginning to uncover striking and unexpected heterogeneity in gene expression at the level of translation.

\section{Outline}

1 Introduction

2 Breaking the single-molecule barrier for live-cell imaging of translation

3 Amplifying live-cell single mRNA translation signals with repeat-epitope tags

4 Limitations of repeat-epitope tags for translation imaging

5 Accessory tags for imaging single mRNA translation in living cells

6 Tagging endogenous proteins with repeatepitope tags

7 Counting ribosomes in polysomes with repeat-epitope tags

8 Quantifying translation elongation with repeat-epitope tags
9 Quantifying translation initiation with repeatepitope tags

10 Quantifying polysome mobility and shape with repeat-epitope tags

11 Emerging consensus in the field of live-cell imaging of single RNA translation

12 Heterogeneity in single mRNA translation efficiency

13 Heterogeneity in ribosome movement along single mRNA

14 Heterogeneity in the localization and mobility of translation sites

15 Outlook

References

Editors: Thomas R. Cech, Joan A. Steitz, and John F. Atkins

Additional Perspectives on RNA Worlds available at www.cshperspectives.org

Copyright (C) 2018 Cold Spring Harbor Laboratory Press; all rights reserved; doi: 10.1101/cshperspect.a032078 


\section{INTRODUCTION}

The translation of messenger RNA (mRNA) into protein is a defining feature of life. All cells must tightly regulate translation to ensure proteins are correctly synthesized in the right place, at the right levels, and at the right time. This allows cells to quickly establish and maintain specific phenotypes in response to internal and external cues as well as environmental pressures (Sonenberg and Hinnebusch 2009; Buxbaum et al. 2015).

Given the dynamic nature of translation, the ability to quantify precisely when, where, and with what kinetics individual mRNA are translated in living cells and organisms provides the most direct means of dissecting complex translational regulatory mechanisms. Although bulk (Ingolia 2014) and single-cell analyses (Han et al. 2014) can provide exquisite snapshots of the average translational state of a specific type of transcript, their lack of spatiotemporal resolution makes it difficult to detect and monitor translational dynamics and heterogeneity at the single mRNA level. This leaves many basic questions about the nature of single mRNA expression unresolved (Chao et al. 2012). First, it remains unclear how heterogeneity in the translation of genetically identical transcripts is established. A variety of posttranscriptional mechanisms are implicated, including acquisition of higher-order structure (Babendure et al. 2006; Wen et al. 2008) and/or chemical modifications (Simms et al. 2014; Hoernes et al. 2016), binding of regulatory factors (Wu et al. 2015; Simsek and Barna 2017), and localization to subcellular structures (Jung et al. 2014; Buxbaum et al. 2015). However, without the ability to image the translation of individual mRNA, distinguishing these factors is extremely difficult. Second, it remains unclear how an individual mRNA is expressed over time (Chao et al. 2012). Is translation constitutive or bursty, localized or homogenous, motored or diffusive? How quickly does translation shut down or start up in response to environmental changes? To what extent does translation dynamics contribute to mRNA decay? Single-molecule imaging of mRNA translation in living cells provides the most direct route to answering these and many other related questions.

In what follows, we describe how technological advances in protein tagging and single-molecule imaging are beginning to revolutionize our understanding of single mRNA translation in living cells. We begin by reviewing breakthroughs in the field, followed by an in-depth discussion of recent applications of repeat-epitope tags. Our emphasis will be on the underlying tagging and imaging technologies and how they synergize to enable the quantification of ribosomal densities, translation initiation and elongation rates, and translation site mobility and higherorder structure (Fig. 1).

\section{BREAKING THE SINGLE-MOLECULE BARRIER FOR LIVE-CELL IMAGING OF TRANSLATION}

The quest to image protein expression in living cells has driven a tremendous amount of research and technology development, particularly in the field of fluorescence microscopy. A milestone was the advent of the green fluorescent protein (GFP), which made it possible to tag and monitor the expression and dynamics of any protein of interest in vivo (Giepmans et al. 2006; Chalfie 2009). Although GFP works tremendously well for most studies, it is not well suited for single-molecule imaging of mRNA translation. This is because GFP takes too long to mature and fluoresce to capture the translation process in realtime (Chao et al. 2012). Furthermore, GFP is not particularly bright or photostable, so it is difficult to see a single GFP fluorophore, even after the lights come on.

To get around this problem, researchers have turned to fluorophores that light up faster than GFP. The Venus fluorescent protein, for example, lights up just minutes after being translated in bacteria. This property, together with the low copy number of proteins in Escherichia coli, made it possible to break the live-cell single-molecule barrier for imaging mRNA translation in living cells (Yu et al. 2006). To achieve this remarkable feat, Venus was fused to a membrane-anchoring protein (serine chemoreceptor protein [Tsr]) and inserted into the native lacZ locus. Expression of Venus was then monitored intermittently between repeated intentional photobleaches of the entire cell. Single Tsr-Venus proteins anchored to the cell membrane could be detected above background by fluorescence immobilization. This revealed that translation occurred in random and uncorrelated bursts, with around one burst per cell cycle and around four Tsr-Venus proteins translated per burst.

Since this initial report, follow-up studies have used Venus fluorescence to quantify translation kinetics in living neurons with single-molecule resolution (Tatavarty et al. 2012; Ifrim et al. 2015; Ströhl et al. 2017). The first of these used cyanine 5-labeled uridine triphosphate (Cy5-UTP) to label mRNA encoding ARC (activity-regulated cytoskeletalassociated protein) and FMRP (fragile X mental retardation protein) tagged with Venus (Tatavarty et al. 2012). Labeled mRNA, introduced to neurons by injection, clustered together and defined the positions of neuronal granules. After whole-cell photobleaching was performed to remove preexisting mature Venus, local translation events were detected by the appearance and rapid single-step photobleaching of individual Venus molecules. A map was then generated showing the positions of all Venus appearance events, which revealed strong clustering near neuronal granules. Like in E. coli, translation was found to be bursty, with multiple translation events occurring over a relatively 

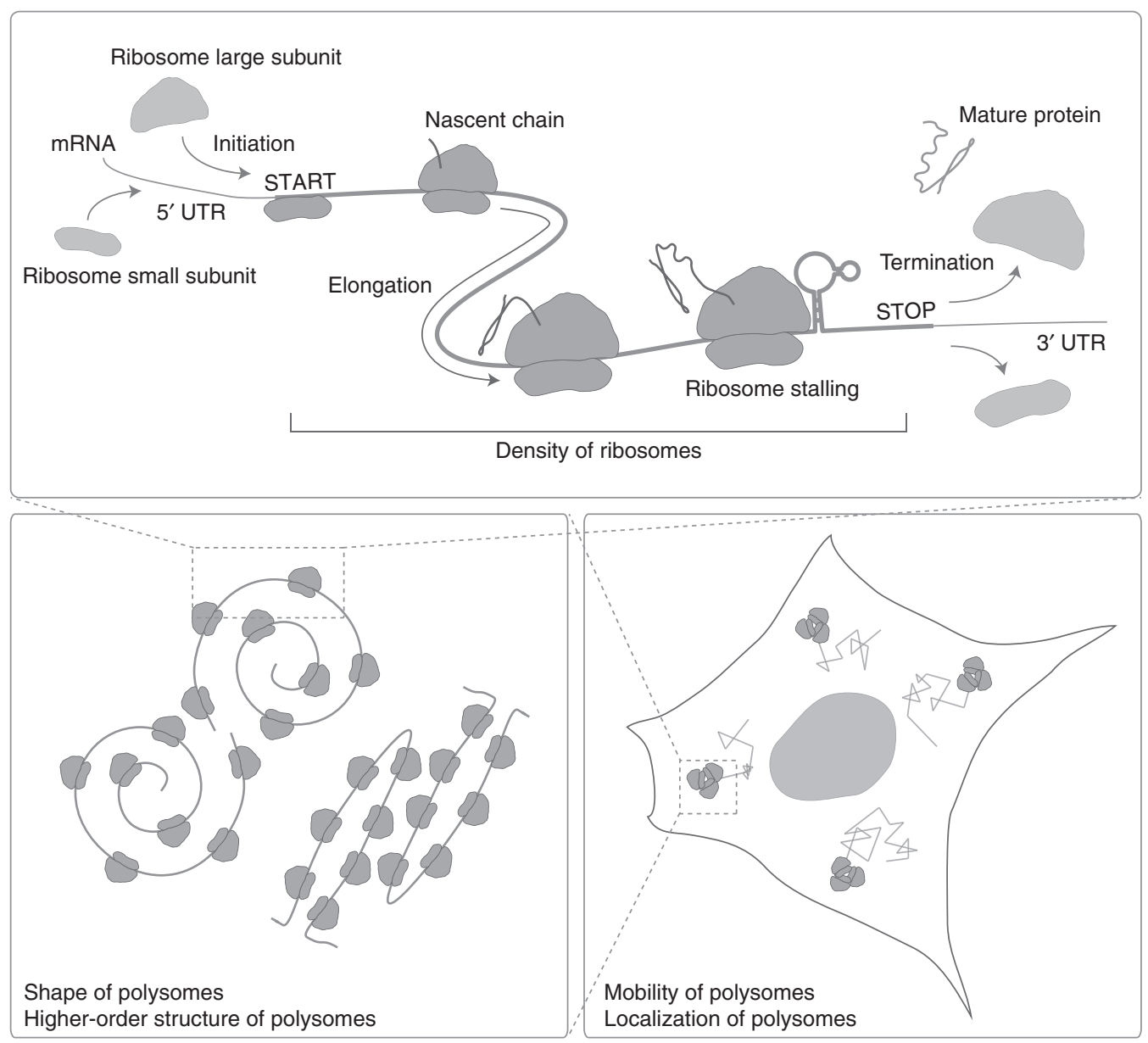

Figure.1. Quantifying messenger RNA (mRNA) translation at the single-molecule level. Many mechanistic details of mRNA translation can now be measured in living cells. (Top) The rates of ribosome initiation, nascent chain elongation, ribosome stalling, and translation termination can be measured, as can the density of ribosomes within polysomes. (Bottom) Polysome shape, higher-order structure, mobility, and localization can also be measured.

short time. Here, bursts were defined as brief periods of high Venus translation, but strictly speaking, it is not possible to know if bursts originated from multiple mRNA in a single granule or from a single mRNA. As burst duration and fluorophore maturation times were convolved, the exact length of an individual burst was difficult to determine. Roughly, bursts were estimated to last around one to two minutes, shorter than Venus maturation times. In the two later studies using Venus in neurons, a similar strategy was used to show that the translation of immobilized VenusPSD-95 (postsynaptic density protein 95) in neuronal dendrites and spines is dysregulated in fragile $\mathrm{X}$ syndrome and that synchronous translation of multiple $\beta$-actin transcripts is initiated by Netrin-1 (Ströhl et al. 2017).

A limitation of single-molecule studies of translation based on the synthesis of Venus (or any other fluorescent fusion protein) are the requirements that (1) nascent proteins be relatively immobile on the timescale of fluorophore maturation and (2) whole-cell photobleaching is required, which could have deleterious side effects (Dobrucki et al. 2007). Photoblinking of Venus also complicates the analysis. A powerful alternative strategy is to shift focus away from the main protein product and instead focus on the impact of translation. For example, as ribosomes elongate, they strip proteins off the coding sequence of mRNA. This effect is beautifully exploited by the TRICK (translation RNA imaging by coat protein knockoff) assay to detect the first round of mRNA translation (Halstead et al. 2015). Specifically, multiple fluorescent probes bound to the coding sequence of an mRNA are irreversibly stripped off by the first ribosome that translates through the mRNA. This loss of signal can then be used to distinguish translated from untranslated mRNA (Chao and Lionnet 2018). One disadvantage of the TRICK assay is that multiple rounds of translation cannot be monitored, because the signal is lost after the first round. However, a unique advantage is that TRICK 
only requires an RNA-binding fluorescent probe. With the advent of clustered regularly interspaced short palindromic repeats (CRISPR)-associated protein 13 (Cas13) (Abudayyeh et al. 2017), Pumilio homology domains (Adamala et al. 2016), and other programmable sequence-specific RNA binders, the TRICK assay could be adapted in the future to detect the translation of endogenous transcripts.

\section{AMPLIFYING LIVE-CELL SINGLE mRNA TRANSLATION SIGNALS WITH REPEAT- EPITOPE TAGS}

Recently, a number of laboratories have independently developed technology to image single mRNA translation dynamics over multiple rounds of translation in living cells (Morisaki et al. 2016; Pichon et al. 2016; Wang et al. 2016; Wu et al. 2016; Yan et al. 2016). As illustrated in Figure 2A, the technologies use fluorescent antibody-like probes to label tagged nascent peptides as they are being translated. Each tag contains a tandem array of short, linear epitopes that are individually bound by probes within seconds of their translation. By repeating epitopes in a single tag, multiple fluorescent probes can label a single nascent polypeptide chain. The signal is further amplified within polysomes, which contain multiple ribosomes translating multiple nascent chains from a single mRNA. The resulting bright signal makes it possible to track single translation sites within living cells for minutes or even hours at a time, not possible with single fluorophores like GFP or Venus.

So far, three epitopes have been used to image single mRNA translation in living cells: the SunTag epitope (EELLSKNYHLENEVARLKK) (Pichon et al. 2016; Wang et al. 2016; Wu et al. 2016; Yan et al. 2016) and the classic FLAG (DYKDDDDK) (Hopp et al. 1988) and HA (hemagglutinin; YPYDVPDYA) (Wilson et al. 1984) epitopes (Morisaki et al. 2016). Within the SunTag (Tanenbaum et al. 2014), individual epitopes are followed by linkers (GSGSG) and the resulting epitope-linker motif is repeated $24 \times$, for a total length of 571 amino acids (aa). Smaller and larger repeats have also been tested, including $4 \times$ (Voigt et al. 2017), $5 \times, 10 \times$ (Yan et al. 2016), 32×, and $56 \times$ repeats (Pichon et al. 2016). In contrast to the uniform distribution of epitopes within SunTags, FLAG and HA epitopes are repeated nonuniformly within "spaghetti monster" tags (Viswanathan et al. 2015). Spaghetti monsters consist of a stable, nonfluorescent GFP-like core structure with strings of epitopes hanging off. In total, spaghetti monsters are $\sim 325$ aa in length, with $3 \times$ epitope repeats at both the amino and carboxyl termini of the core and a $4 \times$ repeat in the inner loop where GFP is commonly split.

Antibody-like probes are required to co-translationally light up the repeat epitopes within the SunTag and spaghetti monsters. In the case of the SunTag, a genetically encoded single-chain variable fragment $(\mathrm{scFv})$ fused to a fluorescent protein like GFP is used ( $\sim 50 \mathrm{kDa}$ total size). The SunTag $\mathrm{scFv}$ was evolved via ribosome display from an anti-general control protein (GCN4) antibody and optimized for stability in the reducing cytoplasm of living cells (Hanes et al. 1998; Tanenbaum et al. 2014). In the case of spaghetti monsters, anti-FLAG or anti-HA antibody fragments (Fabs) conjugated to synthetic dyes like Alexa 488 are used ( $\sim 50 \mathrm{kDa}$ total size). Whereas fluorescent Fab needs to be generated using commercially available kits and parental antibodies, SunTag scFv can be stably expressed in living cells or organisms, a convenient and cost-effective advantage. On the other hand, spaghetti monsters come in many "flavors" for multicolor applications. Besides FLAG and HA, V5, Myc, and OLLAS spaghetti monsters are also available (Viswanathan et al. 2015). Thus, in principle, translation could be imaged in up to five colors with only spaghetti monsters.

\section{LIMITATIONS OF REPEAT-EPITOPE TAGS FOR TRANSLATION IMAGING}

The perfect repeat-epitope tag would light up brightly as soon as translation begins. Two fundamental biophysical constraints prevent this. First, temporal resolution is limited by how long it takes a fluorescent probe to label an epitope. Using microinjection, anti-FLAG Fab were shown to label FLAG spaghetti monster epitopes in $<3 \mathrm{sec}$ (Morisaki et al. 2016). SunTag probes also appear to label their epitopes quickly. When cells were treated with cycloheximide to stall ribosomes, polysome fluorescence did not increase much, suggesting steady state fluorescence had already been reached (Yan et al. 2016). A second factor limiting temporal resolution is epitope length. The longer the epitope, the longer it takes to be translated and detected. The FLAG and HA epitopes are respectively 8 aa and 9 aa in length, whereas the SunTag epitope is 19 aa in length. Provided that probes bind fast enough, the FLAG and HA epitopes can therefore be detected roughly two times faster than SunTag epitopes. However, it may be difficult to detect this difference in practice because of the high background signal from freely diffusing probes and the limited sensitivity of light microscopes.

A major concern of repeat-epitope tags is their large molecular mass and size. When fully labeled, the $24 \times$ SunTag repeat is $1.4 \mathrm{MDa}$, whereas the $10 \times$ spaghetti monster is 0.54 MDa. This extra mass should impact translation. Remarkably, however, measured elongation rates using SunTags and spaghetti monsters largely agree (Table 1). Furthermore, polysome mobility is also largely unaffected by tag size (Table 1). Placing either fewer ( $5 x$ and $10 x$ ) 
A Translation imaging with repeat-epitope tags

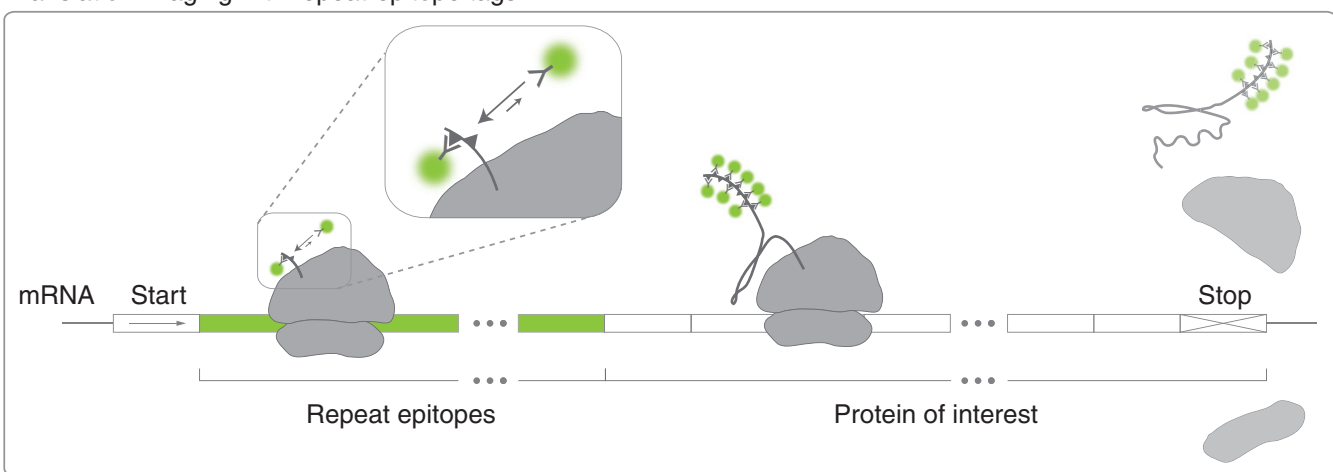

B mRNA tag

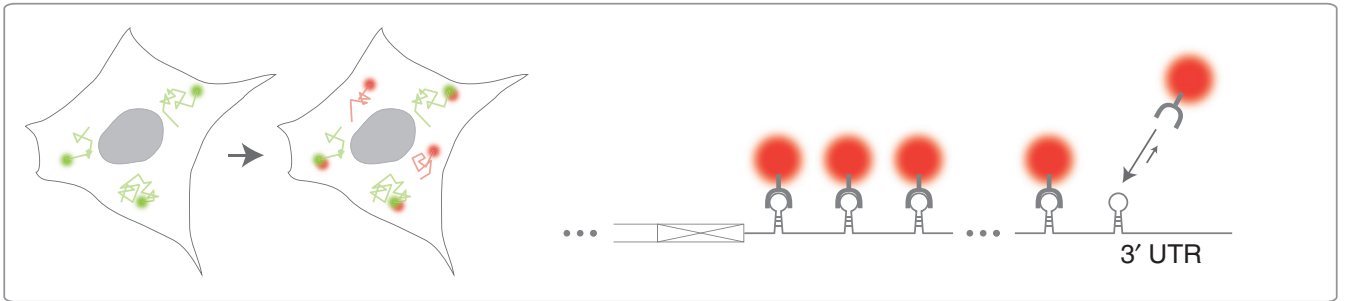

C Immobilization tag

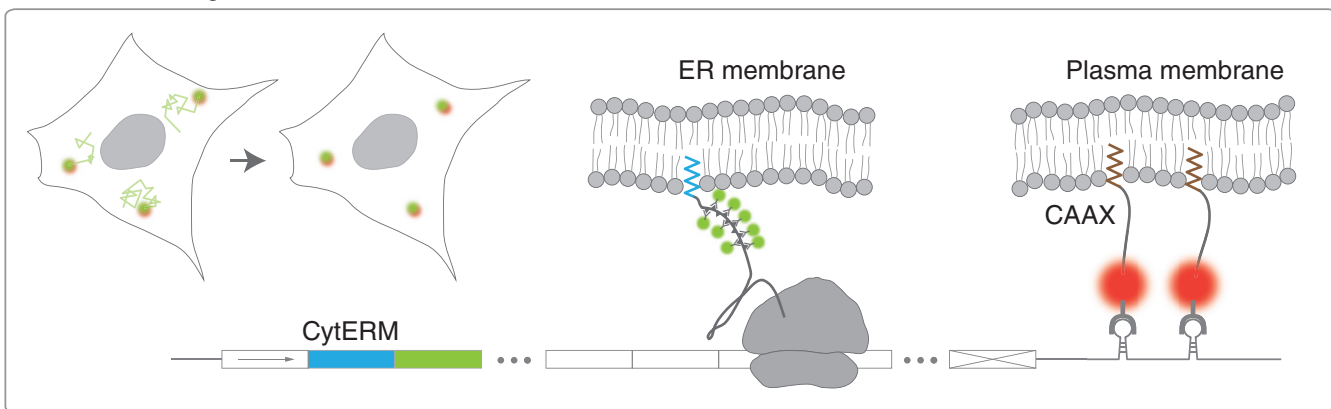

D Protein degron tag

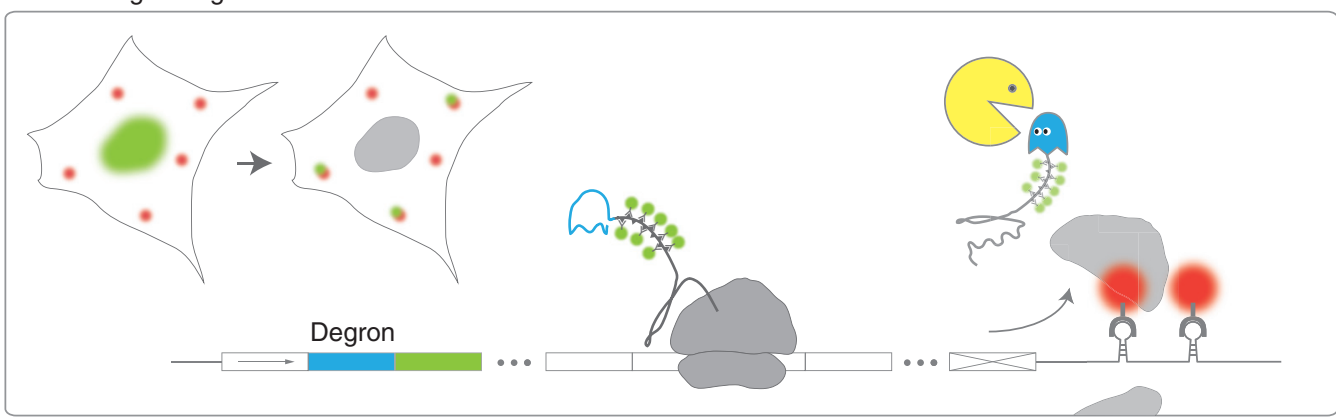

Figure 2. Repeat-epitope and accessory tags for single-molecule imaging of mRNA translation. A schematic showing how repeat-epitope tags are used to visualize single translation sites in living cells. $(A)$ Nascent peptides (small triangles) encoded by repeat epitopes (green mRNA) placed in the amino terminus of a protein of interest are cotranslationally labeled by fluorescent antibody fragments (green spheres). (B) An mRNA tag can facilitate single mRNA translation imaging. Stem loops within the $3^{\prime}$ untranslated region (UTR) of mRNA are co-transcriptionally labeled by coat proteins (red spheres). In combination with a repeat-epitope tag, translation sites are marked in green and red. $(C)$ Immobilization tags can restrict the movement of mRNA so that translation sites can be tracked with less frequent imaging. The cytoplasmic end of an endoplasmic reticulum signal-anchor membrane protein (CytERM) tag (blue) embeds nascent chains into the endoplasmic reticulum (ER) co-translationally, whereas a CAAX domain embeds transcripts with an mRNA tag into the plasma membrane via bound coat proteins (red spheres). (D) A degron (blue mRNA) encoded upstream of the repeat epitopes enhances the degradation of proteins, so nascent chains can be preferentially imaged (rather than mature proteins). 
Downloaded from http://cshperspectives.cshlp.org/ on April 26, 2023 - Published by Cold Spring Harbor Laboratory Press

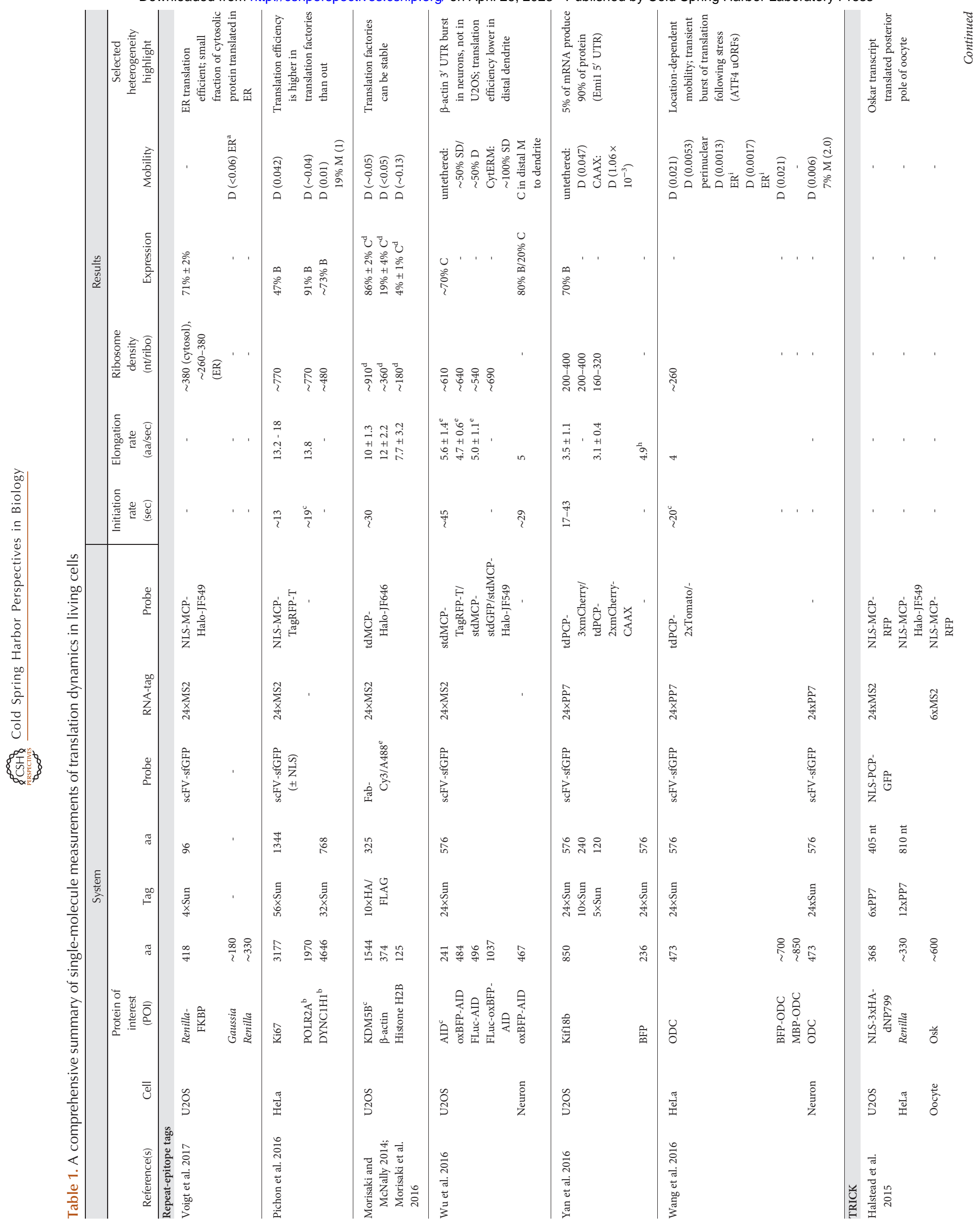




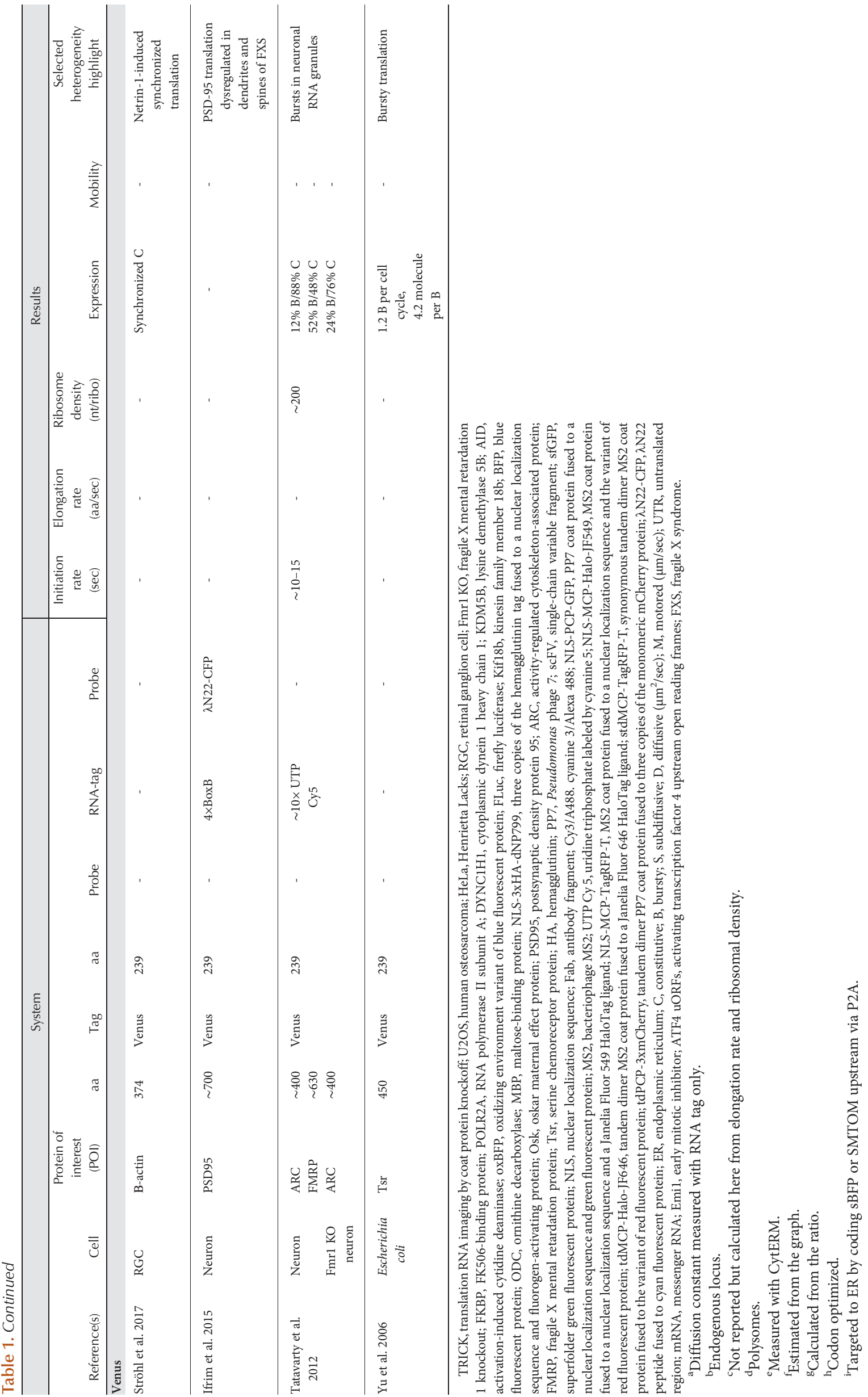


(Yan et al. 2016) or more (32× and 56x) (Pichon et al. 2016) repeats within the SunTag leads to little difference in measured translation kinetics (Table 1). Importantly, reporter proteins tagged with the SunTag were produced at the same levels as untagged proteins (Yan et al. 2016). Together, these data suggest translation sites can cope with the extra mass.

\section{ACCESSORY TAGS FOR IMAGING SINGLE mRNA TRANSLATION IN LIVING CELLS}

In addition to the repeat-epitope tag and probes, accessory tags facilitate the imaging and quantification of single mRNA translation, as summarized in Figure 2. First, a tag for tracking single mRNA is highly recommended. An mRNA tag provides a reference position for translation. Without it, distinguishing translation sites from mature protein or aggregates is difficult. An mRNA tag also makes it possible to (1) count how many mRNA are within a translation site, (2) track bursts of translation on a single mRNA, and (3) determine the fraction of mRNA translating.

So far all groups have used MS2 bacteriophage (Bertrand et al. 1998; Fusco et al. 2003) or Pseudomonas phage 7 (PP7) mRNA tags (Chao et al. 2008) to monitor translation (Fig. 2B). These tags were reviewed recently in Buxbaum et al. 2015. Although extensively tested in living cells and organisms (Lionnet et al. 2011; Lionnet and Singer 2012), mRNA tags may interfere with underlying biology. In yeast, MS2 can artificially stabilize transcripts (Garcia and Parker 2015; Heinrich et al. 2017). Although this may not be a problem for long-lived transcripts in higher eukaryotes, a gentler MS2 tag has been developed to address this problem (Tutucci et al. 2018). An alternative to MS2 or PP7 tags is single molecule RNA FISH (fluorescence in situ hybridization) (Pichon et al. 2016), but this requires fixation.

Another useful accessory for translation imaging is an mRNA immobilization tag (Fig. 2C). The high mobility of mRNA makes it difficult to track their translation kinetics. To get around this issue, mRNA can be tethered to immobile substrates like the endoplasmic reticulum (ER) or the plasma membrane (PM). This makes it possible to image mRNA less frequently and acquire good images without photobleaching. To tether transcripts to the PM, a CAAX domain can be fused to MS2 coat protein (MCP) or PP7 coat protein (PCP) (Fig. 2C) (Yan et al. 2016). To tether to the ER, a cytoplasmic end of an endoplasmic reticulum signal-anchor membrane protein (CytERM) tag can be fused to the amino terminus of the protein of interest (Fig. 2C) (Costantini et al. 2012; Wu et al. 2016). The downside is tethering can alter translation kinetics and prevents the analysis of local translation.
A less essential accessory for translation imaging is a degron tag (Fig. 2D). Degrons are quickly degraded, so any attached proteins do not accumulate. Without a degron, mature proteins sequester probes and eventually prevent translation imaging. Three degrons have been tested so far: ornithine decarboxylase (ODC; 467 aa) (Wang et al. 2016), an auxin-inducible degron (AID; 68 aa) (Wu et al. 2016), and an unstable version of the FK506-binding protein (FKBP; 107 aa) (Voigt et al. 2017). Although degrons are convenient, their degradation may interfere with translation (Hurley et al. 2016). If this is a concern, alternatives to degrons include using an inducible promoter (Yan et al. 2016) or simply imaging soon after transfection (Morisaki et al. 2016), when mature protein levels are low.

\section{TAGGING ENDOGENOUS PROTEINS WITH REPEAT-EPITOPE TAGS}

With the advent of CRISPR-based genome editing, it has become easier to add tags directly to endogenous genes, including repeat epitopes for imaging single mRNA translation (Pichon et al. 2016). The major advantage of this approach is that mRNA and protein arise from their natural, endogenous contexts and are thus maintained at appropriate endogenous levels. The drawback for translation imaging is that endogenous mRNA and protein levels may be high, which could make it hard to detect single translation sites.

To insert $32 \times$ or $56 \times$ amino-terminal SunTag epitopes into endogenous loci, Pichon et al. (2016) used a homologous recombination donor containing a start codon, a selection marker (puromycin), a porcine teschovirus-12A (P2A) peptide cleavage sequence (Kim et al. 2011), and the repeat epitopes, all sandwiched between two homology arms. Properly inserted heterozygous cells were selected using puromycin, and translation beyond the P2A was detected via SunTag probe binding to nascent chains. Two genes were targeted with this strategy, the large subunit of RNA polymerase II (POLR2A) and the heavy chain of dynein 1 (DYNC1H1). Although this strategy is generalizable, both POLR2A (1970 aa) and DYNC1H1 (4646 aa) are relatively large proteins. In combination with the large number of repeat epitopes, tagged transcripts were very long, so translation sites were exceptionally bright. If shorter genes are targeted with fewer repeats, signal-to-noise may become limiting.

Although CRISPR editing is an appealing strategy to examine single mRNA translation kinetics in a nearly endogenous manner, there may be alternatives. Because probes are based on antibodies, endogenous repeat epitopes could in theory be targeted. It has been estimated that roughly one third of all human proteins performing fundamental functions contain tandem repeats (Pellegrini et al. 
1999; Jorda et al. 2010). A well-known example is the heptad repeat in the carboxy-terminal domain of RNA polymerase II, which is repeated around 50 times in humans and for which antibodies and fluorescent Fab already exist (Stasevich et al. 2014). Even if repeats are not available, "programmable" antibodies may someday allow the imaging of any set of epitopes (Ernst and Plückthun 2017), similar to how dCas9 is currently used to light up specific DNA loci in living cells (Chen et al. 2013).

\section{COUNTING RIBOSOMES IN POLYSOMES WITH REPEAT-EPITOPE TAGS}

A strength of imaging single mRNA translation dynamics with repeat-epitope tags is the ability to precisely quantify a number of key biophysical parameters associated with single translation sites, including their ribosomal occupancy, their rates of elongation and initiation, as well as their mobility, localization, and shape (Fig. 1). To calculate how many ribosomes are in polysomes, polysome fluorescence must be normalized to single mature protein fluorescence F. A complication is that ribosomes translating repeatepitope tags on average have translated only half the epitopes. This gives the open reading frame (ORF) an effective length $L^{\prime}=\left(L_{\mathrm{POI}}+0.5 L_{\mathrm{Tag}}\right)$, in which $L_{\mathrm{Tag}}$ is the length of the tag and $L_{\mathrm{POI}}$ is the length of the protein of interest. Then, polysome fluorescence is the product $F \times d \times L^{\prime}$, in which $d$ is the density of ribosomes along the transcript (Pichon et al. 2016; Wang et al. 2016; Yan et al. 2016). If nascent chains cannot be seen above background until a certain number of epitopes have been translated, an additional correction factor makes $L^{\prime}$ smaller (Yan et al. 2016). If tags cannot be observed until they are fully translated or if $L_{\mathrm{POI}}>L_{\mathrm{Tag}}$, then $L^{\prime} \cong L_{\mathrm{POI}}$ (Morisaki et al. 2016).

To accurately determine $F$, sites of mature protein can be identified by their resistance to puromycin and their lack of colocalization with mRNA. Tagging a control protein that does not aggregate or form higher-order structures, such as the maltose-binding protein MBP, may also help (Wang et al. 2016). Alternatively, a second control construct with a single epitope per nascent chain can be imaged (Morisaki et al. 2016). Each fluorophore in a polysome then represents a single nascent chain or ribosome. The number of ribosomes is minimally the total fluorescence divided by the fluorescence of a single fluorophore, the latter being determined unambiguously via single-step photobleaching.

\section{QUANTIFYING TRANSLATION ELONGATION WITH REPEAT-EPITOPE TAGS}

The translation elongation rate is another important parameter that can be quantified from repeat-epitope tag experiments. As shown in Figure 3, this can be performed via ribosomal runoff, fluorescence recovery after photobleaching (FRAP), or fluorescence correlation spectroscopy (FCS). Fundamentally, these all measure the same thing: the fluorescence dwell time at a translation site. To equate the dwell time to the total elongation time, some common assumptions are made. The main technical assumptions are that probes bind epitopes quickly and without interference, as discussed above. In addition, there are a few biological assumptions. First, it is assumed that ribosomes are in steady state so that, on average, when one finishes translation it is replaced by another that starts translation. Second, ribosomes are assumed to always complete translation, so no partial proteins are produced. Third, completed nascent chains are assumed to quickly leave the translation sites. When these assumptions are met, the elongation rate is approximately the effective length $L^{\prime}$ of the ORF divided by the dwell time.

A straightforward way to determine the elongation rate is via ribosomal runoff. The basic idea is that on inhibition of translation initiation, the remaining elongating ribosomes will finish translating one-by-one (i.e., "run off" the mRNA). Natural runoff can be seen if translation is bursty (Pichon et al. 2016; Wu et al. 2016; Yan et al. 2016). Runoff can also be induced by adding the translational inhibitor harringtonine to cell media (Pichon et al. 2016; Wang et al. 2016; Yan et al. 2016). As shown in Figure 3 (top, left), runoff leads to a loss of fluorescence at the translation site in four stages (Pichon et al. 2016; Wang et al. 2016; Yan et al. 2016). The fluorescence starts out in steady state. Harringtonine kicks in during phase II, when fluorescence loss due to runoff is countered by the translation and labeling of new epitopes. Eventually, ribosomes translate past all epitopes, at which point phase III begins and the fluorescence loss is maximal and linear. If many single-transcript runoff curves are averaged, a fourth artifactual phase appears because of heterogeneity (Yan et al. 2016). Fitting phase III of the runoff to a line is an easy way to estimate the elongation rate. The time at which this fitted line extrapolates to zero postharringtonine gives the total dwell or elongation time. Dividing the effective length $L^{\prime}$ of the ORF by the dwell time yields the elongation rate (Wang et al. 2016). Alternatively, more sophisticated models can be used that simultaneously account for the different phases (Pichon et al. 2016; Yan et al. 2016) or translation stochasticity (Wu et al. 2016). If translation is inefficient, single ribosomal runoffs can be observed. Although challenging to capture, the fluorescence signature from these single ribosome events also yields the elongation rate, as illustrated in Figure 3 (bottom, left) (Yan et al. 2016).

A second technique to estimate the elongation rate is FRAP (Fig. 3, top, right). The idea behind FRAP is that 
T. Morisaki and T.J. Stasevich
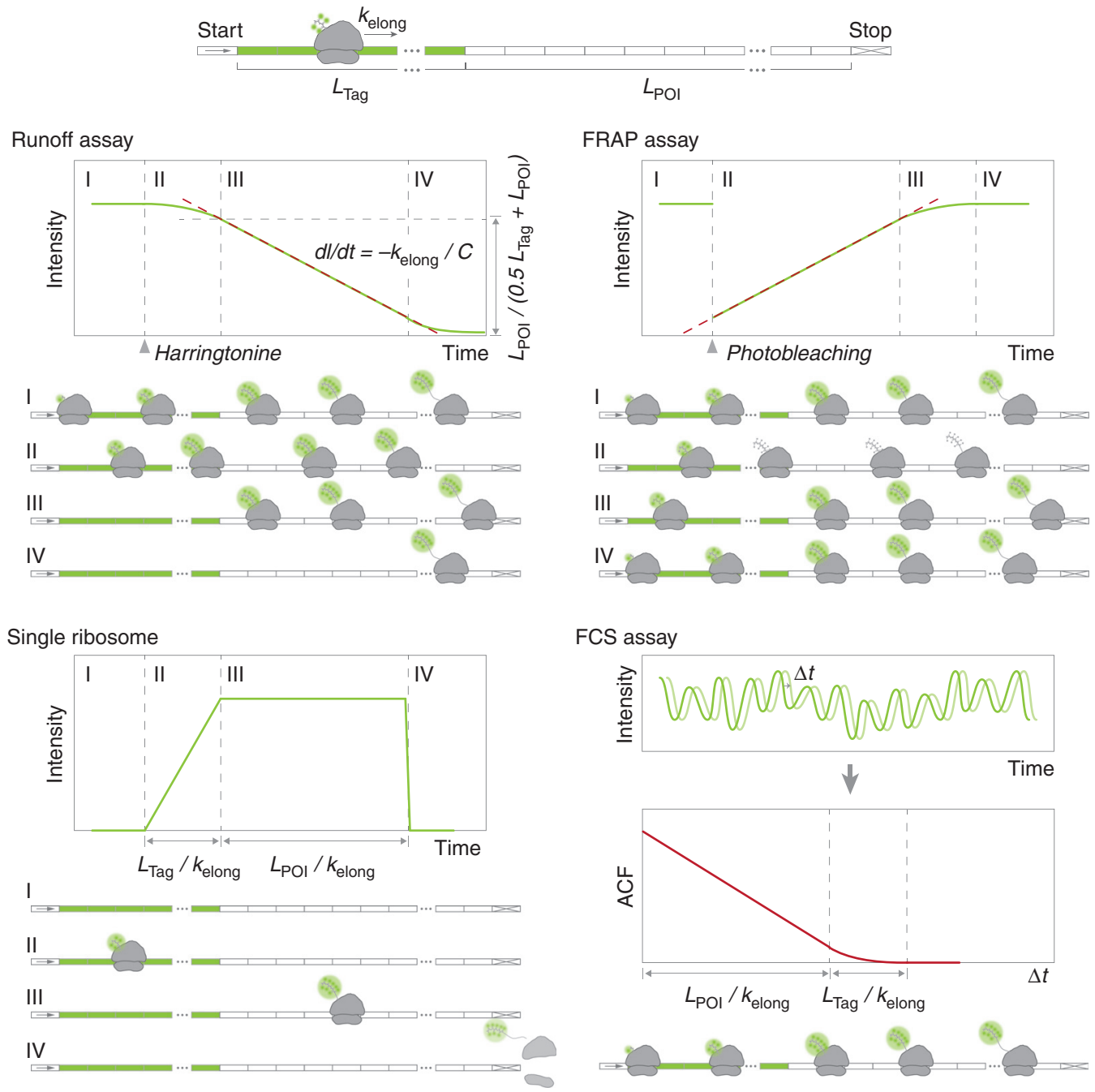

Figure 3. Quantifying translation elongation rates with repeat-epitope tags. The translation elongation rate $k_{\text {elong }}$ can be measured using repeat-epitope tags in multiple ways. Common assumptions are (1) every ribosome that finishes translation is on average replaced by another that starts translation; (2) ribosomes are assumed to always complete translation, so no partial proteins are produced; and (3) completed nascent chains quickly leave the translation sites. $L_{\text {Tag }}$ is the length of the repeat-epitope tag and $L_{\mathrm{POI}}$ is the length of the tagged protein of interest. (Top, left) Addition of the translation initiation inhibitor harringtonine leads to ribosome runoff. During runoff, polysome fluorescence on average drops in four phases (I-IV). The rate of fluorescence decay depends on the elongation rate. (Bottom, left) The fluorescence signal during monosome translation runoff also depends on the elongation rate. (Top, right) Fluorescence recovery after photobleaching (FRAP) of polysomes leads to an average fluorescence recovery that is approximately the inverse of the average polysome runoff fluorescence decay. (Bottom, right) Fluorescence correlation spectroscopy (FCS) at the translation sites produces a decaying autocorrelation function (ACF) that depends on the elongation rate.

nascent chains labeled by photobleached probes will eventually be replaced by newly translated nascent chains labeled by nonphotobleached probes. This requires an additional assumption compared with runoff: The probes should bind irreversibly on the timescale of the FRAP experiment. If not, the fluorescence recovery will be due to probe turnover on single nascent chains rather than nascent chain turnover from translation. Both SunTag probes and
anti-FLAG Fab bind epitopes for tens of minutes at a time, making them suitable for estimating translation dwell times on the order of a few minutes (Morisaki et al. 2016; Yan et al. 2016). Provided ribosomes are in steady state, the fluorescence recovery curve is just the inverse of the runoff curve and can be fit accordingly (Pichon et al. 2016).

Like FRAP, FCS experiments use changes in fluorescence at translation sites to extract translation kinetics 
(Fig. 3, bottom, right) (Stasevich et al. 2010). FCS therefore shares the same common assumptions as FRAP. Unlike FRAP, however, FCS does not require a strong laser pulse to induce an artificial fluctuation in the fluorescence, which could damage cells (Dobrucki et al. 2007). Instead, FCS relies on natural fluctuations that arise from the continual steady state creation and departure of nascent chains. To illustrate, if multiple ribosomes happen to initiate quickly on a single transcript in sequence, then the fluorescence there will get significantly brighter as multiple new nascent chains are translated and labeled by probes. Later, this fluorescence will depart the transcript as the ribosomes terminate in sequence. The time of departure is stochastic, but on average the total dwell time of the fluorescence should be a good approximation for the total elongation time (with all the common assumptions discussed above). If enough natural fluctuations are observed, then the dwell time can be accurately determined from the fluorescence autocorrelation (Morisaki et al. 2016; Wu et al. 2016). The fluorescence autocorrelation can be interpreted as the probability of seeing the same fluorescence at a later time. As translation leads to fluorescence turnover, the autocorrelation generally decays with time, analogous to autocorrelation analysis in the field of transcription (Coulon and Larson 2016). The time at which the autocorrelation drops to zero gives the dwell time of a nascent chain at the mRNA, from which the elongation rate can again be calculated as in FRAP or runoff. More sophisticated stochastic models can also be used to fit the full curve (Wu et al. 2016).

An advantage of FRAP and FCS relative to runoff is they can be performed on any transcript without the need for drugs or the requirement of bursty expression. On the other hand, both FRAP and FCS require care when performing and interpreting data. For example, the intentional FRAP photobleach has many potential pitfalls, including photodamage (Dobrucki et al. 2007), photoinduced cross-linking or unbinding (Heinze et al. 2009), and photoswitching (Mueller et al. 2012; Morisaki and McNally 2014). Likewise, FCS is technically demanding (requiring many hundreds or thousands of individual images), and sensitive to experimental noise from photobleaching, nonhomogeneous illumination, tracking errors, diffusion of free probe, and focusing issues (Coulon and Larson 2016).

\section{QUANTIFYING TRANSLATION INITIATION WITH REPEAT-EPITOPE TAGS}

Once the number of ribosomes in transcripts and the total elongation time are measured, it is relatively straightforward to calculate the translation initiation rate. The calculation again relies on the steady state assumption, in which ribosomes enter and exit translation sites at a constant, balanced rate. Then, the total elongation time divided by the number of ribosomes on the transcript gives the time between ribosomes. Assuming uniform movement along the transcript, this time is equal to the initiation time (i.e., the inverse initiation rate) (Morisaki et al. 2016; Pichon et al. 2016; Wang et al. 2016; Wu et al. 2016; Yan et al. 2016).

\section{QUANTIFYING POLYSOME MOBILITY AND SHAPE WITH REPEAT-EPITOPE TAGS}

Besides ribosome density, initiation, and elongation kinetics, the strong fluorescence of repeat-epitope-tagged nascent chains makes it possible to track them with great precision in living cells and characterize their mobility and shape. To quantify the mobility of mRNAs, mean-squared displacement is a standard metric (Saxton 1996). Four categories of mobility have been observed, in order of increasing dynamics: confined, corralled, diffusive, and motored (Park et al 2010). Confined polysomes are bound to an immobile substrate. Some cutoff is generally required to define "bound." A threshold can be obtained by independently measuring the mobility of the confining structure. For example, the mobility of histone H2B has been used to define chromatin-bound transcription factors (Mazza et al. 2012; Morisaki et al. 2014). A similar approach could be used to define mRNA confinement to the PM or ER using CAAX domains (Hancock et al. 1991; Yan et al. 2016) or CytERM (Costantini et al. 2012; Wu et al. 2016) immobilizers, respectively. In contrast to total confinement, corralled polysomes are able to move about freely within a restricted subcellular volume. The mean-squared displacement for corralled polysomes starts off linear, but eventually plateaus to a peak value that marks the boundaries of the corralled zone. If the restricted volume is the entire cytoplasm, then polysome movement is purely diffusive and the meansquared displacement is linear. Finally, motored or "superdiffusive" polysomes move about in an erratic fashion with brief periods of rapid movement in random directions at roughly constant velocity (Pichon et al. 2016; Wu et al. 2016). The mean squared displacement in this case is described by a curve with an exponent between one (pure diffusion) and two (constant velocity).

To quantify the shape of translation sites encoding repeat-epitope-tagged proteins, the intramolecular fluorescence distribution can be analyzed (Morisaki et al. 2016). As the fluorescence originates from nascent chains labeled by probes, the spatial extent of the chains constrains the spatial resolution of imaging, in addition to diffraction. Because three nucleotides totaling $\sim 1 \mathrm{~nm}$ in length encode a single amino acid $\sim 0.35 \mathrm{~nm}$ in length, the ORF of an mRNA is around three times longer than the nascent chain it encodes when both are stretched out. Including both 
untranslated regions (UTRs), a transcript can therefore be up to an order of magnitude longer than the protein it encodes. To illustrate, the $\beta$-actin transcript is $\sim 370 \mathrm{~nm}$ when spread out (not diffraction-limited with $488 \mathrm{~nm}$ light), whereas the nascent chains would only be 374 aa $\times$ $0.35 \mathrm{~nm} \sim 130 \mathrm{~nm}$ (diffraction-limited). Nascent chain fluorescence, therefore, has the potential to provide subdiffractive structural information about a single translation site if ribosomes are sufficiently spread out within the site.

To accurately capture the intramolecular fluorescence of translation sites, imaging exposure times should be fast compared with the movement of the translation site. Faster exposures will create a better snapshot and prevent artifacts due to movement. Fixation could be used as a movement control as well. Spots may be diffraction-limited, in which case they will appear the same shape and size as images of immobilized 100-nm beads on a coverslip. If so, superresolution can be achieved by fitting the fluorescence distribution to a Gaussian function. The center of the Gaussian distribution function gives the average coordinates of the nascent chains (Morisaki et al. 2016). Deviations from the expected diffraction-limited spot may indicate the translation site is spread out in some manner. If an RNA tag is also included, the distribution of fluorescence within nascent chains can also be compared with the distribution of fluorescence within the RNA tag. This approach was used to show that translation sites composed of two mRNA had dimensions roughly twice the size of single mRNA translation sites, indicating the individual mRNAs retained their spatial independence despite co-movement (Morisaki et al. 2016)

\section{EMERGING CONSENSUS IN THE FIELD OF LIVE- CELL IMAGING OF SINGLE RNA TRANSLATION}

With the advent of repeat-epitope tags, fast-maturing fluorescent proteins like Venus, and RNA-based assays like TRICK (and, more recently, 3'-RNA end accumulation during turnover [TREAT]; Horvathova et al. 2017) to unambiguously spot hard-to-see molecular events in living cells, we expect the number of single-molecule studies of translation to grow significantly in the near future. Given the early state of the field, it is rather remarkable that there is already some consensus in terms of the quantification of translation kinetics. First, single mRNA studies of translation in eukaryotes have found good agreement in measured translation elongation rates. The average is close to $10 \mathrm{aa} /$ $\mathrm{sec}$, with an experimental range between 3 and $18 \mathrm{aa} / \mathrm{sec}$ (Table 1). Whereas this range is in part explained by codon usage (Yan et al. 2016), the overall order-of-magnitude agreement probably reflects underlying biophysical constraints on molecular motors. Consistent with this, $10 \mathrm{aa} / \mathrm{sec}$ corresponds to $1800 \mathrm{nt} / \mathrm{min}$, which is close to what has been measured for transcription elongation by RNA polymerase II (Darzacq et al. 2009; Larson et al. 2011; Stasevich et al. 2014). In the future, it will be interesting to see how tightly coupled transcription and translation elongation rates are for specific genes in eukaryotic cells.

In addition to translation elongation rates, the measured density of ribosomes along active transcripts is also fairly consistent between studies, being one ribosome every 200 to $1000 \mathrm{nt}$. This order-of-magnitude agreement becomes even better when calculating ribosomal initiation rates from the measured ribosomal densities and elongation rates. In this case, all studies agree within a factor of just three, finding between two and five ribosomes initiating per minute on translationally active transcripts (Table 1). Again, this excellent agreement in the initiation rates may reflect some underlying biophysical constraint that limits the number of ribosomes that can initiate in a set amount of time. Alternatively, it could be an artifact of small sample size. More studies will be needed to get a better sampling of the full distribution of ribosome initiation and elongation rates.

\section{HETEROGENEITY IN SINGLE mRNA TRANSLATION EFFICIENCY}

In what follows, we highlight some of the interesting heterogeneity that has so far been uncovered by imaging the translation of single mRNA. As Table 1 summarizes, the heterogeneity takes a variety of forms, including heterogeneity in translation efficiency, ribosome dynamics, and polysome mobility and localization.

One of the most striking observations of single mRNA translation is that individual transcripts can switch between translationally active and inactive states (Table 1). This bursty behavior is likely a universal feature of gene expression, as it has now been observed in both living bacteria $(\mathrm{Yu}$ et al. 2006) and eukaryotes (Tatavarty et al. 2012; Pichon et al. 2016; Wang et al. 2016; Wu et al. 2016; Yan et al. 2016). In contrast to bursty translation, some transcripts appear to be constitutively expressed, i.e., perpetually in an active state in which initiation is always possible (Morisaki et al. 2016). Whereas the line between bursty and constitutive expression is somewhat gray, it seems clear that more abundant proteins are more likely to be translated in a constitutive fashion. For example, there are hundreds of thousands of RNA polymerase II proteins per cell (Kimura et al. 1999; Stasevich et al. 2014). Not surprisingly then, POL2R, the RNA polymerase II transcript, is constitutively translated at very high levels, with upward of $90 \%$ of mRNA being translated at any given time. In contrast, just $40 \%-70 \%$ of transcripts encoding components of the molecular motors dynein and kinesin are translated at any given time. These 
bursty transcripts stochastically switch between active and inactive states on the timescale of minutes to hours. These examples suggest that burstiness might be genetically encoded, but this is certainly not always the case. A counterexample is the $\beta$-actin transcript, which is constitutively expressed in human osteosarcoma (U2OS) cells (Morisaki et al. 2016; Wu et al. 2016), but bursty in neurons (Wu et al. 2016; Ströhl et al. 2017). Transcripts specifically in neurons seem especially prone to bursty behavior (Tatavarty et al. 2012; Ifrim et al. 2015; Wang et al. 2016; Wu et al. 2016; Ströhl et al. 2017). This could reflect a requirement to shut down or ramp up translation in a localized manner (Holt and Schuman 2013). Thus, both genetic and posttranscriptional factors dictate the expression patterns of individual transcripts in vivo.

An interesting consequence of heterogenous mRNA translation efficiency is that a relatively small subset of mRNA may be responsible for the vast majority of proteins produced in a cell. A good illustration of this point is the Emil 5' UTR, which has a long isoform with several upstream ORFs (Yan et al. 2016). The upstream ORFs sequester ribosomes. Because of this, only $20 \%$ of reporter mRNA harboring the long $5^{\prime}$-UTR isoform were observed to be actively translated, most with low efficiency. Remarkably, a small subset of these active mRNA was robustly translated, creating $>50 \%$ of the final protein product. Thus, even among a presumably genetically identical pool of mRNA, translation may be heterogeneous on multiple levels, with a minor subset of a small fraction producing most proteins against the odds. How general this phenomenon is and what specific factors facilitate it remain open questions.

\section{HETEROGENEITY IN RIBOSOME MOVEMENT ALONG SINGLE mRNA}

Accurate measurements of translation elongation rates at the single mRNA level make it possible to directly address questions of codon usage and ribosome stalling. The most direct test of codon usage to date was an examination of the blue fluorescent protein BFP (Yan et al. 2016). A codonoptimized version of this construct elongated at an average rate of $4.9 \mathrm{aa} / \mathrm{sec}$, whereas an unoptimized version (encoding Kif18b) had a rate of just $3.1 \mathrm{aa} / \mathrm{sec}$. Although the optimized and unoptimized transcripts have different average rates, it is important to point out that there may be great variability within each pool. For example, FCS experiments on genetically identical individual transcripts showed some could be translated over twice as fast as others (Morisaki et al. 2016). This is an emerging theme: Variation in the translation kinetics within a pool of presumably identical transcripts can be as great or even greater than the average variation seen between two distinct pools of transcripts.
Other evidence for heterogeneity in ribosome movement along single RNA comes from ribosomal runoff assays (Pichon et al. 2016; Wang et al. 2016; Yan et al. 2016). Here, individual runoff curves rarely look like the mean, with some fast and others quite slow. Also, there may be a small subset of transcripts that shut down slowly, if at all (Yan et al. 2016). Presumably these transcripts have ribosomes that have either slowed down significantly or altogether stalled. The slowdown was attributed to damaged mRNA, as the percentage of slow runoff transcripts could be increased by treatment with 4NQO (4-nitroquilone-1oxide) to oxidize RNA nucleotides (Yan et al. 2016). These data provide evidence that specific posttranscriptional modifications to nucleotides can significantly impact the elongation rate of ribosomes along transcripts.

Finally, genetically encoded pause sites can be directly inserted into transcripts to measure heterogeneity of ribosomal movement along single mRNA. The stress-related factor $\mathrm{Xbp} 1$ has a genetically encoded pause that helps target mRNA to the membrane (Ingolia et al. 2011; Yanagitani et al. 2011). When inserted into a reporter SunTag construct, polysome runoffs were observed to be nonlinear. This nonlinearity indicates heterogeneity in elongation among individual ribosomes within single polysomes. Also, a subset of polysomes showed a delayed runoff in response to harringtonine. This was interpreted as a traffic jam of ribosomes that was suddenly relieved.

\section{HETEROGENEITY IN THE LOCALIZATION AND MOBILITY OF TRANSLATION SITES}

One possible explanation for the translational heterogeneity seen among transcripts in single cells is their local subcellular environment. Local translation is thought to occur in special regions of the cell where translation efficiency is enhanced. For example, it was recently shown that individual transcripts translated in the ER have more ribosomes than those translated in the cytoplasm (Voigt et al. 2017), suggesting the efficiency of translation is higher in the ER. Furthermore, interaction with the ER is not limited exclusively to secreted proteins, as transcripts encoding cytoplasmic proteins also appear to occasionally associate with the ER when being translated. Considering the position of the ER around the cell nucleus, these interactions are likely responsible for the generally slower mobility of perinuclear translation sites observed (Wang et al. 2016).

In addition to localization within specific organelles, active transcripts also appear to occasionally colocalize (and comove) with one another to form higher-order structures. Such "translation factories" have been observed for both exogenous (Morisaki et al. 2016) and endogenous constructs (Pichon et al. 2016). These sites probably have higher 
local concentrations of translation factors and are therefore translated more efficiently. Consistent with this, endogenously tagged dynein transcripts within factories were translated with $90 \%$ efficiency, compared with just $70 \%$ outside factories. Importantly, the factories are translation-dependent, as treatment with a rapamycin inhibitor, which decreases translation initiation efficiency by two- to threefold (Thoreen et al. 2012), led to a significant loss of mRNA within the factories (Pichon et al. 2016). Similarly, treatment with puromycin results in the breakup of factories (TJ Stasevich, T Morisaki, K Lyon, pers. observation). It remains unclear what molecular mechanisms promote these higherorder structures. Future work is therefore needed to definitively prove the sites are bona fide translation factories rather than just transient associations by chance.

The motored transport of transcripts to specific sites is one mechanism by which local translation may be established. Motored transport of both actively translating and inactive single transcripts has been observed in living eukaryotic cells (Pichon et al. 2016; Wang et al. 2016; Wu et al. 2016). The motored motion is from 1 to $3 \mu \mathrm{m} / \mathrm{sec}$ and is typically sporadic, being interspersed between purely diffusive movements (Pichon et al. 2016; Wang et al. 2016). In neurons, motored transport is an efficient way to localize transcripts to dendrites and axons that may be physically separated from the nucleus by distances as large as a meter. Consistent with this, ARC transcripts harboring a $3^{\prime}$-UTR "zip code" that targets them to dendrites were motored with much higher probability in neurons than in HeLa cells (Wang et al. 2016). The motored movement was preferentially in an anterograde direction, resulting in a buildup of ARC polysomes in dendrites (Wang et al. 2016). Once in dendrites, ARC transcripts are translated in granules that undergo bursts of translation in an action potential-dependent manner (Tatavarty et al. 2012). This spatiotemporal regulation of translation is thought to be important for the integration of electrical signals into long-term phenotypic neuronal changes that are necessary for memory and learning. The observation that ARC and other transcripts can be motored when actively translated goes against the conventional view that transcripts are silenced until they reach their destination. For example, the tracking of single oskar transcripts during Drosophila development with the TRICK assay showed that translation does not occur until they reach the posterior poles of the oocyte (Halstead et al. 2015). As more transcripts are studied in different cellular contexts, it will be interesting to see how common active translation is during motored transport. The newer data suggest that transcripts may by default be in an active state amenable to translation, but the active state is switched off in specific contexts when bursty expression or complete silencing is required.

\section{OUTLOOK}

The last 20 years has seen tremendous progress in visualizing and quantifying single mRNA translation dynamics in living cells. Over the next 20 years, what can we expect from this technology? There is certainly plenty of room for improvement. In terms of repeat-epitope tag technology, more and smaller probes are needed for multiplexing and reduced invasiveness. Potential candidates that could replace $\mathrm{scFv}$ and Fab include chromobodies/nanobodies (Rothbauer et al. 2006), monobodies (Koide and Koide 2007), affibodies (Gao et al. 2011), DARPins (Plückthun 2015), thermobodies (Zhao et al. 2016), and split fluorescent reporters (Kamiyama et al. 2016). An ideal probe might be a single amino acid, which could bring live-cell imaging of translation on par with in vitro imaging (Perez and Gonzalez 2011), which already enjoys single-codon resolution. How this will be achieved is not yet clear, but promising starting points are noncanonical amino acids. Recently, Cy dyes were site-specifically incorporated into proteins in Xenopus laevis oocytes as noncanonical amino acids (Leisle et al. 2016), so it is not a stretch to imagine this being performed with fluorescently tagged mRNA to monitor translation dynamics. The ultimate goal would be in situ sequencing of protein synthesis in vivo. If achievable, this would place us directly behind the ribosome, so that we could see what it sees and fully comprehend its extraordinary behavior in translational regulation.

\section{ACKNOWLEDGMENTS}

We thank Amanda Koch and Charlotte Cialek for their helpful discussion and comments. T.J.S. and T.M. are supported by the W.M. Keck Foundation and the National Institutes of Health (NIH) grant number R35GM119728. T.J.S. is also supported by funds from the Boettcher Foundation's Webb-Waring Biomedical Research Program.

\section{REFERENCES}

* Reference is also in this collection.

Abudayyeh OO, Gootenberg JS, Essletzbichler P, Han S, Joung J, Belanto JJ, Verdine V, Cox DBT, Kellner MJ, Regev A, et al. 2017. RNA targeting with CRISPR-Cas13. Nature 550: 280-284.

Adamala KP, Martin-Alarcon DA, Boyden ES. 2016. Programmable RNA-binding protein composed of repeats of a single modular unit. Proc Natl Acad Sci 113: E2579-E2588.

Babendure JR, Babendure JL, Ding J-H, Tsien RY. 2006. Control of mammalian translation by mRNA structure near caps. RNA 12: 851-861.

Bertrand E, Chartrand P, Schaefer M, Shenoy SM, Singer RH, Long RM. 1998. Localization of ASH1 mRNA particles in living yeast. Mol Cell 2: 437-445.

Buxbaum AR, Haimovich G, Singer RH. 2015. In the right place at the right time: Visualizing and understanding mRNA localization. Nat Rev Mol Cell Biol 16: 95-109. 
Chalfie M. 2009. GFP: Lighting up life. Proc Natl Acad Sci 106: 1007310080.

* Chao JA, Lionnet T. 2018. Imaging the life and death of mRNAs in single cells. Cold Spring Harb Perspect Biol 10: a032086.

Chao JA, Patskovsky Y, Almo SC, Singer RH. 2008. Structural basis for the coevolution of a viral RNA-protein complex. Nat Struct Mol Biol 15: 103-105.

Chao JA, Yoon YJ, Singer RH. 2012. Imaging translation in single cells using fluorescent microscopy. Cold Spring Harb Perspect Biol 4: a012310.

Chen B, Gilbert LA, Cimini BA, Schnitzbauer J, Zhang W, Li G-W, Park J, Blackburn EH, Weissman JS, Qi LS, et al. 2013. Dynamic imaging of genomic loci in living human cells by an optimized CRISPR/Cas system. Cell 155: 1479-1491.

Costantini LM, Fossati M, Francolini M, Snapp EL. 2012. Assessing the tendency of fluorescent proteins to oligomerize under physiologic conditions. Traffic 13: 643-649.

Coulon A, Larson DR. 2016. Chapter Seven-Fluctuation analysis: Dissecting transcriptional kinetics with signal theory. In Methods in enzymology (eds. Filonov GS, Jaffrey SR), Vol. 572, Visualizing RNA dynamics in the cell, pp. 159-191. Academic, Cambridge, MA.

Darzacq X, Yao J, Larson DR, Causse SZ, Bosanac L, de Turris V, Ruda VM, Lionnet T, Zenklusen D, Guglielmi B, et al. 2009. Imaging transcription in living cells. Annu Rev Biophys 38: 173-196.

Dobrucki JW, Feret D, Noatynska A. 2007. Scattering of exciting light by live cells in fluorescence confocal imaging: Phototoxic effects and relevance for FRAP studies. Biophys J 93: 1778-1786.

Ernst P, Plückthun A. 2017. Advances in the design and engineering of peptide-binding repeat proteins. Biol Chem 398: 23-29.

Fusco D, Accornero N, Lavoie B, Shenoy SM, Blanchard J-M, Singer RH, Bertrand E. 2003. Single mRNA molecules demonstrate probabilistic movement in living mammalian cells. Curr Biol CB 13: 161-167.

Gao J, Chen K, Miao Z, Ren G, Chen X, Gambhir SS, Cheng Z. 2011. Affibody-based nanoprobes for HER2-expressing cell and tumor imaging. Biomaterials 32: 2141-2148.

Garcia JF, Parker R. 2015. MS2 coat proteins bound to yeast mRNAs block $5^{\prime}$ to $3^{\prime}$ degradation and trap mRNA decay products: Implications for the localization of mRNAs by MS2-MCP system. RNA NYN 21: 1393-1395.

Giepmans BNG, Adams SR, Ellisman MH, Tsien RY. 2006. The fluorescent toolbox for assessing protein location and function. Science 312: 217-224.

Halstead JM, Lionnet T, Wilbertz JH, Wippich F, Ephrussi A, Singer RH, Chao JA. 2015. An RNA biosensor for imaging the first round of translation from single cells to living animals. Science 347: 1367-1671.

Han K, Jaimovich A, Dey G, Ruggero D, Meyuhas O, Sonenberg N, Meyer T. 2014. Parallel measurement of dynamic changes in translation rates in single cells. Nat Methods 11: 86-93.

Hancock JF, Cadwallader K, Paterson H, Marshall CJ. 1991. A CAAX or a CAAL motif and a second signal are sufficient for plasma membrane targeting of ras proteins. EMBO J 10: 4033-4039.

Hanes J, Jermutus L, Weber-Bornhauser S, Bosshard HR, Plückthun A. 1998. Ribosome display efficiently selects and evolves high-affinity antibodies in vitro from immune libraries. Proc Natl Acad Sci 95: 14130-14135.

Heinrich S, Sidler CL, Azzalin CM, Weis K. 2017. Stem-loop RNA labeling can affect nuclear and cytoplasmic mRNA processing. RNA 23: 134-141.

Heinze KG, Costantino S, De Koninck P, Wiseman PW. 2009. Beyond photobleaching, laser illumination unbinds fluorescent proteins. JPhys Chem B 113: 5225-5233.

Hoernes TP, Hüttenhofer A, Erlacher MD. 2016. mRNA modifications: Dynamic regulators of gene expression? RNA Biol 13: 760-765.

Holt CE, Schuman EM. 2013. The central dogma decentralized: New perspectives on RNA function and local translation in neurons. Neuron 80: $648-657$
Hopp TP, Prickett KS, Price VL, Libby RT, March CJ, Cerretti DP, Urdal DL, Conlon PJ. 1988. A short polypeptide marker sequence useful for recombinant protein identification and purification. Nat Biotechnol 6: 1204-1210.

Horvathova I, Voigt F, Kotrys AV, Zhan Y, Artus-Revel CG, Eglinger J, Stadler MB, Giorgetti L, Chao JA. 2017. The dynamics of mRNA turnover revealed by single-molecule imaging in single cells. Mol Cell 68: 615-625.e9.

Hurley JM, Loros JJ, Dunlap JC. 2016. Circadian oscillators: Around the transcription-translation feedback loop and on to output. Trends Biochem Sci 41: 834-846.

Ifrim MF, Williams KR, Bassell GJ. 2015. Single-molecule imaging of PSD-95 mRNA translation in dendrites and its dysregulation in a mouse model of fragile X syndrome. J Neurosci 35: 7116-7130.

Ingolia NT. 2014. Ribosome profiling: New views of translation, from single codons to genome scale. Nat Rev Genet 15: 205-213.

Ingolia NT, Lareau LF, Weissman JS. 2011. Ribosome profiling of mouse embryonic stem cells reveals the complexity and dynamics of mammalian proteomes. Cell 147: 789-802.

Jorda J, Xue B, Uversky VN, Kajava AV. 2010. Protein tandem repeatsThe more perfect, the less structured. FEBS J 277: 2673-2682.

Jung H, Gkogkas CG, Sonenberg N, Holt CE. 2014. Remote control of gene function by local translation. Cell 157: 26-40.

Kamiyama D, Sekine S, Barsi-Rhyne B, Hu J, Chen B, Gilbert LA, Ishikawa H, Leonetti MD, Marshall WF, Weissman JS, et al. 2016. Versatile protein tagging in cells with split fluorescent protein. Nat Commun 7: 11046.

Kim JH, Lee S-R, Li L-H, Park H-J, Park J-H, Lee KY, Kim M-K, Shin BA, Choi S-Y. 2011. High cleavage efficiency of a 2A peptide derived from porcine teschovirus-1 in human cell lines, zebrafish and mice. PLoS One 6: e18556.

Kimura H, Tao Y, Roeder RG, Cook PR. 1999. Quantitation of RNA polymerase II and its transcription factors in an HeLa cell: Little soluble holoenzyme but significant amounts of polymerases attached to the nuclear substructure. Mol Cell Biol 19: 5383-5392.

Koide A, Koide S. 2007. Monobodies: Antibody mimics based on the scaffold of the fibronectin type III domain. In Methods in molecular biology (ed. Walker JM), Vol. 352, Protein engineering protocols (eds. Arndt KM, Müller KM), pp. 95-109. Humana, Totowa, NJ.

Larson DR, Zenklusen D, Wu B, Chao JA, Singer RH. 2011. Real-time observation of transcription initiation and elongation on an endogenous yeast gene. Science 332: 475-478.

Leisle L, Chadda R, Lueck JD, Infield DT, Galpin JD, Krishnamani V, Robertson JL, Ahern CA. 2016. Cellular encoding of Cy dyes for singlemolecule imaging. eLife 5: pii: e19088.

Lionnet T, Singer RH. 2012. Transcription goes digital. EMBO Rep 13: 313-321.

Lionnet T, Czaplinski K, Darzacq X, Shav-Tal Y, Wells AL, Chao JA, Park HY, de Turris V, Lopez-Jones M, Singer RH. 2011. A transgenic mouse for in vivo detection of endogenous labeled mRNA. Nat Methods 8: $165-170$.

Mazza D, Abernathy A, Golob N, Morisaki T, McNally JG. 2012. A benchmark for chromatin binding measurements in live cells. Nucleic Acids Res 40: e119.

Morisaki T, McNally JG. 2014. Photoswitching-free FRAP analysis with a genetically encoded fluorescent tag. PLoS One 9: e107730.

Morisaki T, Müller WG, Golob N, Mazza D, McNally JG. 2014. Singlemolecule analysis of transcription factor binding at transcription sites in live cells. Nat Commun 5: 4456.

Morisaki T, Lyon K, DeLuca KF, DeLuca JG, English BP, Zhang Z, Lavis LD, Grimm JB, Viswanathan S, Looger LL, et al. 2016. Real-time quantification of single RNA translation dynamics in living cells. Science 352: $1425-1429$.

Mueller F, Morisaki T, Mazza D, McNally JG. 2012. Minimizing the impact of photoswitching of fluorescent proteins on FRAP analysis. Biophys J 102: 1656-1665. 
Park HY, Buxbaum AR, Singer RH. 2010. Chapter 18-Single mRNA tracking in live cells. In Methods in enzymology (ed. Walter NG), Vol. 472, Single molecule tools: Fluorescence based approaches, Part A, pp. 387-406, Academic, Cambridge, MA.

Pellegrini M, Marcotte EM, Yeates TO. 1999. A fast algorithm for genome-wide analysis of proteins with repeated sequences. Proteins 35: 440-446.

Perez CE, Gonzalez RL. 2011. In vitro and in vivo single-molecule fluorescence imaging of ribosome-catalyzed protein synthesis. Curr Opin Chem Biol 15: 853-863.

Pichon X, Bastide A, Safieddine A, Chouaib R, Samacoits A, Basyuk E, Peter M, Mueller F, Bertrand E. 2016. Visualization of single endogenous polysomes reveals the dynamics of translation in live human cells. J Cell Biol 214: 769-781.

Plückthun A. 2015. Designed ankyrin repeat proteins (DARPins): Binding proteins for research, diagnostics, and therapy. Annu Rev Pharmacol Toxicol 55: 489-511.

Rothbauer U, Zolghadr K, Tillib S, Nowak D, Schermelleh L, Gahl A, Backmann N, Conrath K, Muyldermans S, Cardoso MC, et al. 2006. Targeting and tracing antigens in live cells with fluorescent nanobodies. Nat Methods 3: 887-889.

Saxton MJ. 1996. Anomalous diffusion due to binding: A Monte Carlo study. Biophys J 70: 1250-1262.

Simms CL, Hudson BH, Mosior JW, Rangwala AS, Zaher HS. 2014. An active role for the ribosome in determining the fate of oxidized mRNA. Cell Rep 9: 1256-1264.

Simsek D, Barna M. 2017. An emerging role for the ribosome as a nexus for post-translational modifications. Curr Opin Cell Biol 45: 92-101.

Sonenberg N, Hinnebusch AG. 2009. Regulation of translation initiation in eukaryotes: Mechanisms and biological targets. Cell 136: 731-745.

Stasevich TJ, Mueller F, Michelman-Ribeiro A, Rosales T, Knutson JR, McNally JG. 2010. Cross-validating FRAP and FCS to quantify the impact of photobleaching on in vivo binding estimates. Biophys J 99: 3093-3101.

Stasevich TJ, Hayashi-Takanaka Y, Sato Y, Maehara K, Ohkawa Y, SakataSogawa K, Tokunaga M, Nagase T, Nozaki N, McNally JG, et al. 2014. Regulation of RNA polymerase II activation by histone acetylation in single living cells. Nature 516: 272-275.

Ströhl F, Lin JQ, Laine RF, Wong HH-W, Urbančič V, Cagnetta R, Holt CE, Kaminski CF. 2017. Single molecule translation imaging visualizes the dynamics of local $\beta$-actin synthesis in retinal axons. Sci Rep 7: 709 .
Tanenbaum ME, Gilbert LA, Qi LS, Weissman JS, Vale RD. 2014. A protein-tagging system for signal amplification in gene expression and fluorescence imaging. Cell 159: 635-646.

Tatavarty V, Ifrim MF, Levin M, Korza G, Barbarese E, Yu J, Carson JH. 2012. Single-molecule imaging of translational output from individual RNA granules in neurons. Mol Biol Cell 23: 918-929.

Thoreen CC, Chantranupong L, Keys HR, Wang T, Gray NS, Sabatini DM. 2012. A unifying model for mTORC1-mediated regulation of mRNA translation. Nature 485: 109-113.

Tutucci E, Vera M, Biswas J, Garcia J, Parker R, Singer RH. 2018. An improved MS2 system for accurate reporting of the mRNA life cycle. Nat Methods 15: 81-89.

Viswanathan S, Williams ME, Bloss EB, Stasevich TJ, Speer CM, Nern A, Pfeiffer BD, Hooks BM, Li W-P, English BP, et al. 2015. High-performance probes for light and electron microscopy. Nat Methods 12: 568576.

Voigt F, Zhang H, Cui XA, Triebold D, Liu AX, Eglinger J, Lee ES, Chao JA, Palazzo AF. 2017. Single-molecule quantification of translationdependent association of mRNAs with the endoplasmic reticulum. Cell Rep 21: 3740-3753.

Wang C, Han B, Zhou R, Zhuang X. 2016. Real-time imaging of translation on single mRNA transcripts in live cells. Cell 165: 990-1001.

Wen J-D, Lancaster L, Hodges C, Zeri A-C, Yoshimura SH, Noller HF, Bustamante C, Tinoco I. 2008. Following translation by single ribosomes one codon at a time. Nature 452: 598-603.

Wilson IA, Niman HL, Houghten RA, Cherenson AR, Connolly ML, Lerner RA. 1984. The structure of an antigenic determinant in a protein. Cell 37: 767-778.

Wu B, Buxbaum AR, Katz ZB, Yoon YJ, Singer RH. 2015. Quantifying protein-mRNA interactions in single live cells. Cell 162: 211-220.

Wu B, Eliscovich C, Yoon YJ, Singer RH. 2016. Translation dynamics of single mRNAs in live cells and neurons. Science 352: 1430-1435.

Yan X, Hoek TA, Vale RD, Tanenbaum ME. 2016. Dynamics of translation of single mRNA molecules in vivo. Cell 165: 976-989.

Yanagitani K, Kimata Y, Kadokura H, Kohno K. 2011. Translational pausing ensures membrane targeting and cytoplasmic splicing of XBP1u mRNA. Science 331: 586-589.

Yu J, Xiao J, Ren X, Lao K, Xie XS. 2006. Probing gene expression in live cells, one protein molecule at a time. Science 311: 1600-1603.

Zhao N, Schmitt MA, Fisk JD. 2016. Phage display selection of tight specific binding variants from a hyperthermostable Sso7d scaffold protein library. FEBS J 283: 1351-1367. 


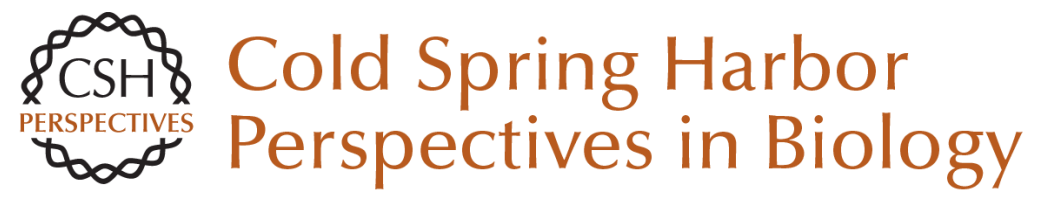

\section{Quantifying Single mRNA Translation Kinetics in Living Cells}

Tatsuya Morisaki and Timothy J. Stasevich

Cold Spring Harb Perspect Biol 2018; doi: 10.1101/cshperspect.a032078

\section{Subject Collection RNA Worlds}

Alternate RNA Structures

Marie Teng-Pei Wu and Victoria D'Souza

Approaches for Understanding the Mechanisms

of Long Noncoding RNA Regulation of Gene

Expression

Patrick McDonel and Mitchell Guttman

Principles and Practices of Hybridization Capture

Experiments to Study Long Noncoding RNAs That

Act on Chromatin

Matthew D. Simon and Martin Machyna

Linking RNA Sequence, Structure, and Function

on Massively Parallel High-Throughput

Sequencers

Sarah K. Denny and William J. Greenleaf

Extensions, Extra Factors, and Extreme

Complexity: Ribosomal Structures Provide

Insights into Eukaryotic Translation

Melanie Weisser and Nenad Ban

Nascent RNA and the Coordination of Splicing with Transcription

Karla M. Neugebauer

Combining Mass Spectrometry (MS) and Nuclear Magnetic Resonance (NMR) Spectroscopy for Integrative Structural Biology of Protein-RNA Complexes

Alexander Leitner, Georg Dorn and Frédéric H.-T. Allain
Structural Biology of Telomerase

Yaqiang Wang, Lukas Susac and Juli Feigon

Structural Insights into Nuclear pre-mRNA

Splicing in Higher Eukaryotes

Berthold Kastner, Cindy L. Will, Holger Stark, et al.

What Are 3' UTRs Doing?

Christine Mayr

Single-Molecule Analysis of Reverse

Transcriptase Enzymes

Linnea I. Jansson and Michael D. Stone

CRISPR Tools for Systematic Studies of RNA

Regulation

Jesse Engreitz, Omar Abudayyeh, Jonathan

Gootenberg, et al.

Relating Structure and Dynamics in RNA Biology Kevin P. Larsen, Junhong Choi, Arjun Prabhakar, et al.

Beyond DNA and RNA: The Expanding Toolbox of Synthetic Genetics

Alexander I. Taylor, Gillian Houlihan and Philipp Holliger

For additional articles in this collection, see http://cshperspectives.cshlp.org/cgi/collection/

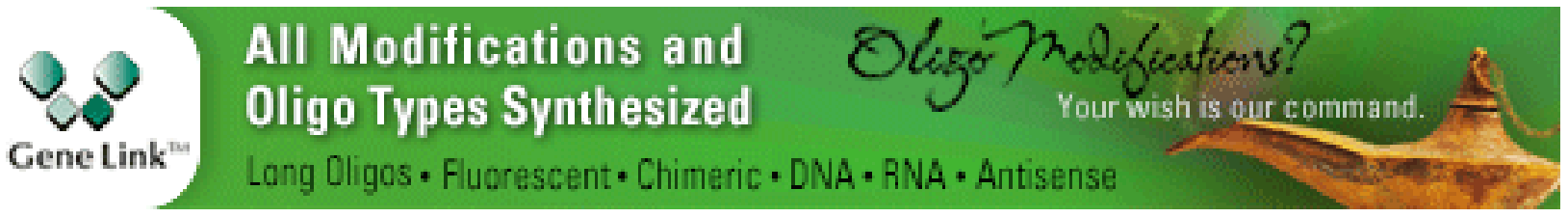

Copyright @ 2018 Cold Spring Harbor Laboratory Press; all rights reserved 
Discovering and Mapping the Modified Nucleotides That Comprise the Epitranscriptome of mRNA

Bastian Linder and Samie R. Jaffrey
Structural Basis of Nuclear pre-mRNA Splicing:

\section{Lessons from Yeast}

Clemens Plaschka, Andrew J. Newman and Kiyoshi Nagai

For additional articles in this collection, see http://cshperspectives.cshlp.org/cgi/collection/

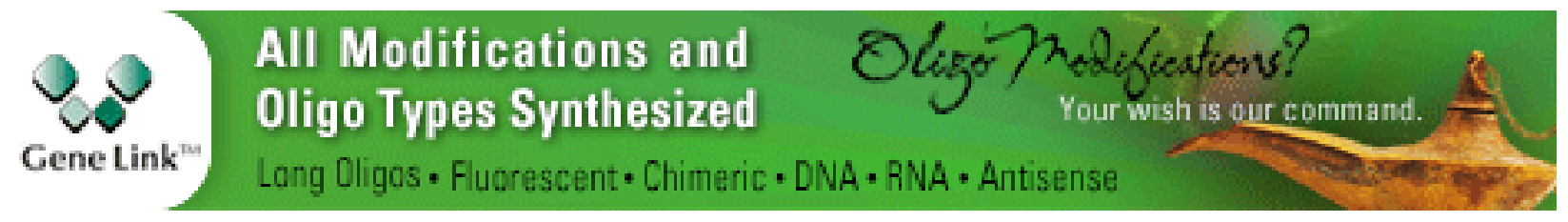

Copyright (C) 2018 Cold Spring Harbor Laboratory Press; all rights reserved 